\title{
MINIREVIEW
}

\section{The mathematics of sexual attraction}

\author{
José A Feijó* \\ See research article: http://www.biomedcentral.com/1471-2229/10/32
}

\begin{abstract}
Pollen tubes follow attractants secreted by the ovules. In a recent paper in BMC Plant Biology, Stewman and colleagues have quantified the parameters of this attraction and used them to calibrate a mathematical model that reproduces the process and enables predictions on the nature of the female attractant and the mechanisms of the male response.
\end{abstract}

Darwin referred to the rapid success of flowering plants in evolution as an 'abominable mystery'. A great deal of this success relies on the peculiar ways of sexual reproduction that they have evolved. The facts that delight us and spark our curiosity about the sexual behavior of animals, in fact the essence of many novels attraction, deception, chemistry, male competition, female selection, abortion, death and sacrifice - take place just below our noses every time we appreciate the fine scent of a flower.

The lovers, usually invisible to the eye, are the male and female gametophytes, the pollen grain and the embryo sac; the plot occurs inside the female reproductive organs, collectively known as the pistil (Figure 1). The final act involves the irresistible attraction to the ovule through specific molecules, leading inevitably to the altruistic self-sacrifice of the pollen tube cell, which explodes to deliver the sperm cells to the embryo sac.

Ever since Linnaeus, the process has intrigued biologists, and although the fundamental basis of the interactions were understood in the 19th century by researchers such as Robert Brown, Giovanni Battista Amici, Darwin and Sergei Nawaschin [1], the molecular nature of this fatal attraction is only now beginning to be understood [2]. Mathematics is now being used to

\footnotetext{
*Correspondence: jfeijo@fcul.pt
}

Instituto Gulbenkian de Ciência, P-2780-156 Oeiras, Portugal and Universidade de Lisboa, Faculdade de Ciências, Depto. Biologia Vegetal, Campo Grande C2, P-1749-016 Lisboa, Portugal investigate the problem for the first time, in a groundbreaking article recently published in BMC Plant Biology [3]. In this study, Stewman and colleagues use a semi-vivo system to better quantify the nature and range of the ovule's attractants. More importantly, they applied a stochastic mathematical model of whole-cell motility to pollen tube growth. This sort of model has previously been used with success to describe chemotaxis in various eukaryotic cells, such as leukocytes and Listeria. When calibrated with the new experimental data, the model enabled predictions to be made about the size and physical features of the attractant. The results suggest that the growth features observed greatly enhance the attraction efficiency of the ovules.

\section{The complex anatomy of a fatal attraction}

Most of the beauty that flowers may have for us through their combinations of colors, shapes and scents have, from a developmental point of view, only one purpose: attracting insects and other animals through deception to perform pollination. Reproduction occurs when a pollen grain lands on the receptive surface of pistil, the stigma (Figure 1), whether it arrives there through a sophisticated relationship between insects and orchids or through the mere dispersion of grass pollen by the wind. There, the word 'chemistry' has a literal meaning as well as a metaphorical one, because the bonding between the specialized outer layers of the cell walls of the pollen and the outer stigma cells has been calculated to be stronger than the strongest industrial superglue [1]. Many receptors and ligands come into place to assure proper recognition, and if the match is compatible, the stigma cells nurture the highly dehydrated pollen grains by providing them with water and nutrients, allowing germination. The pollen tube then grows out of the pollen grain in an extreme example of polarized, apical cell growth that results from an unusual set of cell features [4]. The pollen tube is one of the fastest growing cells in nature, and encodes a very specialized transcriptome for cell signaling and communication, which makes them efficient stimulus-perception machines [1]. But how and why these cell-cell interactions take place and navigate the pollen tube so precisely to 


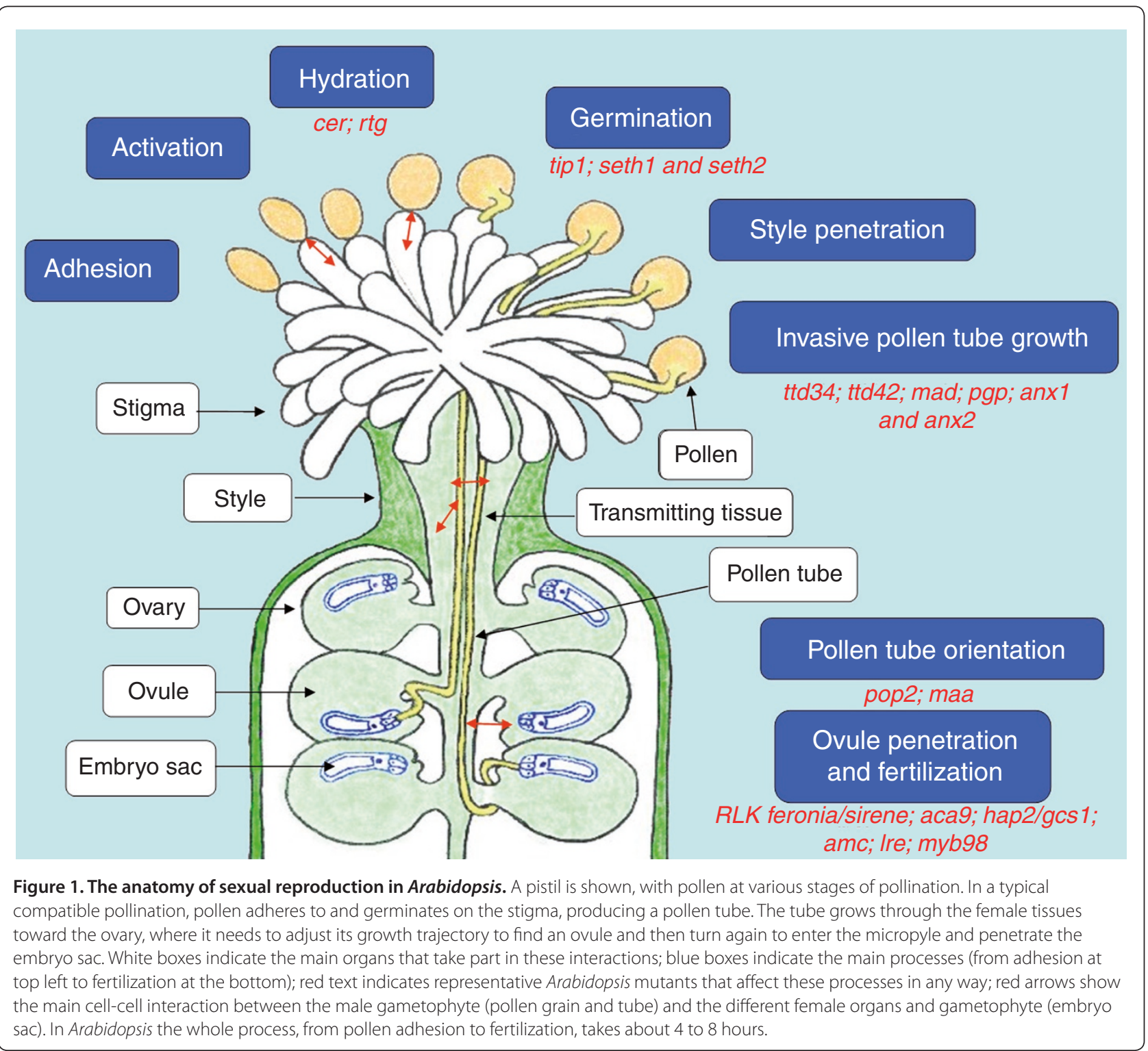

target the ovule's entrance - the micropyle - are still matters of debate.

These events all take place deep inside the pistil tissues, and it has only recently been possible to image them directly by means of two-photon microscopy [5]. In the decades since the pioneering work of Rosen, Mascarenhas and others, evidence has accumulated that a combination of chemotropic molecules is probably needed for some of the steps. However, for most of the path (from adhesion to entry into the ovary; Figure 1), the anatomical arrangement of the tissues seems to be sufficient to mechanically provide a limited freedom for tube growth. The physical and chemical features of the tissues that make direct contact with pollen tubes (lipids, water, glycosylated proteins, and so on) provide the rest of the signals $[1,6]$. With the advent of genetics, a number of screens in Arabidopsis isolated mutations for most steps of this so-called progamic phase of reproduction (a representative list of mutants for each step is shown in red in Figure 1). Some of these mutants have provided evidence for long-range targeting, with signaling occurring over perhaps as far as $500 \mu \mathrm{m}$. The list of genes involved is likely to get significantly larger as new screening methods reveal dozens of male- and femalespecific mutations, especially in Arabidopsis [7], but so far none has brought us close to the specific chemotaxis molecule(s).

\section{Of gradients and explosive discharges}

Curiously enough, answers to the question of the chemotaxis molecule's identity came from classical experimental embryology. For a long time, various teams 
have been developing partially artificial fertilization schemes, for either fundamental or applied reasons [6]. In most of these schemes, pollen is allowed to germinate in the stigma, but the style is excised and the pollen tubes are allowed to grow out of the style into an artificial medium. This semi-vivo method has more recently been combined with isolated ovules to test the hypothesis of whether the system is sufficient to provide attraction to the pollen tube; this would validate the existence of gradients of attractants secreted from the ovules. Such a system was first shown to work in the succulent plant Gasteria [8] and more recently in the model species Arabidopsis [9], the lily Lilium [10] and maize [11]. But none of these has been as powerful and informative as the system developed by Higashiyama and colleagues using the wishbone flower, Torenia fournieri [6]. Starting from the anatomical observation that in this species the female gametophyte - the embryo sac - is naked and exposed without any surrounding tissue, these authors [6] developed a series of experiments that showed for the first time the explosive discharge of the pollen tube inside the ovule and identified the synergid cells (Figure 2) as the source of the diffusing attractant signal [6]. In a tourde-force of proteomics, the same authors recently isolated the first proteins to be shown to be specifically involved in the attractant signal: small, defensin-like,

Figure 2. Ovule attraction and the chemotaxis of pollen tube growth. (a) Pollen tube guidance precedes double fertilization in flowering plants. A pollen tube carrying two sperm cells leaves the placenta to grow along the funiculus (the foot of the ovule) into the micropyle (the entrance of the ovule) following gradients generated by the maternal tissues of the ovule and by the female gametophyte. An embryo sac contains the egg apparatus (egg cell and two synergid cells), the central cell with two polar nuclei, and three antipodal cells. It is usually surrounded by a supportive tissue - the nucellus - and two layers of protective tissue - the inner and outer integuments. In Torenia the nucellus is disintegrated, generating a naked egg apparatus at the micropylar region. Adapted from [1 1]. (b,c) Semi-vivo growth system in Arabidopsis. Pollen is germinated in the stigma, but the style is cut (top in (b)) and cocultivated with dissected ovules (bottom in (b)). When coming out of the style, pollen tubes grow in the surface of a semi-solid agar medium, and eventually target the micropyles of the ovules (c). If penetration is achieved, the contents of the tubes are discharged inside one synergid; if the system is carried out with pollen tubes (arrows in (c)) labeled with green fluorescent protein, the moment of fertilization is visible by fluorescence (arrowheads in (c)), and ovules can be scored in terms of successful attraction. The scale bars represent $100 \mathrm{~mm}$. Adapted from [9]. (d) Depiction of the angles used in the analysis of pollen tube turning made by Stewman et al. [3]. These angles indicate how much the pollen tube would have to turn to take the most direct path toward the micropyle $\left(q_{m p}\right)$, and describe the new direction chosen by the pollen tube in response to the gradient $\left(q_{t i p}\right)$. These quantitative data were then gathered for various incubation periods to deduce the nature and effect of the gradient produced by the diffusion of an attractant from the ovule's micropyle.

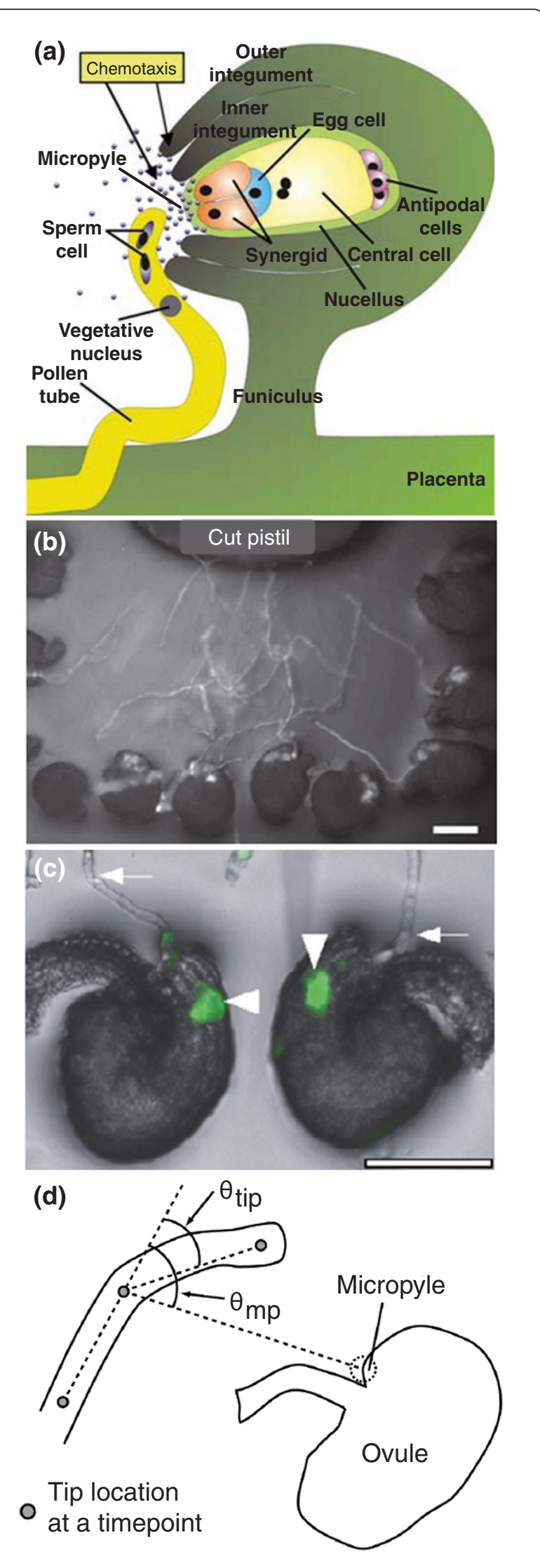


cysteine-rich peptides called LUREs [12]. A model of pollen tube attraction by diffusion of molecules from the micropyle now seems to be well established (Figure 2a). If comparisons can be drawn from the animal field [11], many more classes of proteins are expected to be described, as the proteomics effort is only just starting and many screening efforts are getting under way [7].

\section{Attraction through gradients: where there is a ligand there must be a receptor}

The development of a suitable semi-vivo system of fertilization for Arabidopsis (Figure 2b,c) has not only enabled the use of genetics, it has also been used to investigate the physical nature of the diffusing molecule in Arabidopsis, as it has in Torenia [8]. Stewman et al. [3] have now carefully analyzed the growth curvature angles of Torenia pollen tubes (Figure 2d) in various experimental conditions, namely with different incubation times of the ovules (presumably corresponding to different levels of a standing gradient), and could thus determine important quantitative parameters of the attraction process. First, they found that the gradient action could extend a distance of 100 to $150 \mathrm{~mm}$ [3], a distance significantly longer than had been previously thought $[6,9]$ or than had been experimentally tested with artificial gradients of isolated molecules $[2,12]$. This distance estimate probably means that various types of molecules with different ranges and actions come together to produce the biological reaction. There is no doubt that species specificity must be assigned by geneencoded proteins, and that the isolated LUREs and the Zea mays egg apparatus 1 protein (ZmEA1) both produce positive tropic effects. However, the evidence seems to implicate many other non-specific, small, diffusible chemicals, such as ions or even the signaling gas nitric oxide (NO) [10]. In fact, the involvement of NO is not a surprise, because various behaviors shown by pollen tubes when NO concentration is disturbed seem to indicate a slowing down of growth of the pollen tube as it approaches the diffusion source; these effects fit with those found in the model of Stewman and colleagues [3].

The apparent simplicity of pollen tube growth is leading to various approaches to mathematical modeling of its main features. Pollen tubes have been successfully described through mechanical or geometrical approaches [13]. Looking from a different angle, the prominence of the signaling systems of pollen tube growth was recently modeled by assuming that soluble $N$-ethylmaleimide sensitive factor attachment protein receptors (SNAREs) and small signaling GTPases are the main effectors of growth [14].

Stewman et al. [3] took a step further and focused on the formal properties of the system as shown from the kinetic parameters precisely derived from the semi-vivo system. The problem of gradient sensing is not trivial, and the authors [3] followed the strategy of building on stochastic whole-cell motility models, which basically assume that pollen tubes can sense a difference in the fraction of the receptors bound to an attractant and change their growth depending on this fraction. One prediction of the model was that a slower growth rate inside a standing gradient of attractant (which is assumed to exist from the isotropic diffusion of the attractant from the ovule) would greatly enhance the ability of pollen tubes to successfully target ovules, a prediction that they could statistically validate with their experiments [3]. Furthermore, the model describes the observed patterns of random and directed growth observed during growth of pollen tubes in vitro.

Although the assumption of a purely formal mathematical description of any biological phenomenon may lead to purely phenomenological descriptions of limited experimental value, the complexity of the system and the number of different cellular components that seem to be fundamental for growth [4] make these approaches highly informative, or even fundamental, to understanding the mechanistic basis of the macroscopic response of the system. For example, this model [3] may help us to understand how pollen tubes couple external guidance cues with intracellular ion gradients or other known cell steering mechanisms. In addition, the model assumes that there are at least two patches of receptors, which are separated in the pollen tube. Although this assumption says nothing about what the receptors are, it does suggest that a minimal model of a sensor with a strict apical point location would not work; instead, it favors a membrane or cytosolic spatially segregate or a receptor that stretches across (at least) the diameter of the tube. The model also assumes that the change in concentration across the tip of the tube is much less than the average concentration at the tip. Despite being affected by the limitation of the two-dimensional modeling performed [3], this is a strong quantitative prediction that can be validated when searching for novel molecules that might fit the profile. And how does slowing down of the tubes take place near the micropyle? NO, for example, is known for slowing down growth rates [10], and the formulation of the model does, in fact, allow for multi-factorial interactions affecting the growth process. Importantly, this mathematical formulation may allow discrimination of different effects during experimental procedures based both on the deviation of the growth angle and/or the relationship of the growth rate to successful targeting.

As with many other mathematical approaches to complex biological behavior, this new model from Stewman et al. [3] raises more questions than answers. But the fact that new approaches are contributing to a 
precise experimental description of the system $[2,11,12]$ may make mathematical modeling an important tool for testing and selecting candidate molecules that may fit the in vivo biological profile of the final step of plant sexual attraction.

\section{Acknowledgements}

I thank Leonor Boavida for drawing and compiling Figure 1. Financial support for my laboratory is provided by FCT grants PTDC/BIA-BCM/108044/2008 and PTDC/QUI/64339/06.

\section{Published: 29 March 2010}

\section{References}

1. Boavida, L, Becker, JD, Vieira, AM, Feijó, JA: Gametophyte interaction and sexual reproduction: how plants make a zygote. Int J Dev Bio/ 2005, 49:615-632

2. Dresselhaus T, Márton ML: Micropylar pollen tube guidance and burst: adapted from defense mechanisms? Curr Opin Plant Biol 2009, 12:773-780.

3. Stewman SF, Jones-Rhoades M, Bhimalapuram P, Tchernookov M, Preuss $D$, Dinner AR: Mechanistic insights from a quantitative analysis of pollen tube guidance. BMC Plant Biol 2010, 10:32.

4. Michard E, Alves RF, Feijó JA: The role of ion fluxes in polarized cell growth: the pollen tube as an experimental paradigm. Int J Dev Biol 2009, 53: 1609-1622

5. Cheung AY, Boavida LC, Aggarwal M, Wu HM, Feijó JA: The pollen tube journey in the pistil. Imaging the in vivo process by two-photon microscopy. J Exp Bot, in press.

6. Higashiyama T, Hamamura Y: Gametophytic pollen tube guidance. Sex Plant Reprod 2008, 21:17-26.
7. Boavida LC, Shuai B, Yu HJ, Pagnussat GC, Sundaresan V, McCormick S: A collection of Ds insertional mutants associated with defects in male gametophyte development and function in Arabidopsis thaliana. Genetics 2009, 181:1369-1385.

8. Willemse MTM, Plyushch TA, Reinders MC: In vitro micropylar penetration of the pollen tube in the ovule of Gasteria verrucosa. Plant Science 1995, 108: 201-208.

9. Palanivelu R, Preuss D: Distinct short-range ovule signals attract or repel Arabidopsis thaliana pollen tubes in vitro. BMC Plant Biol 2006, 6:7.

10. Prado, AM, Colaço, R, Moreno, N, Silva, AM, Feijó JA: Targeting to ovules is dependent on nitric oxide signalling. Mol Plant 2008, 1:703-714.

11. Márton M, Dresselhaus T: A comparison of early molecular fertilization mechanisms in animals and flowering plants. Sex Plant Reprod 2008, 21:37-52.

12. Okuda S, Tsutsui H, Shiina K, Sprunck S, Takeuchi H, Yui R, Kasahara RD, Hamamura Y, Mizukami A, Susaki D, Kawano N, Sakakibara T, Namiki S, Itoh K, Otsuka K, Matsuzaki M, Nozaki H, Kuroiwa T, Nakano A, Kanaoka MM, Dresselhaus T, Sasaki N, Higashiyama T: Defensin-like polypeptide LUREs are pollen tube attractants secreted from synergid cells. Nature 2009 458:357-361.

13. Geitmann A: How to shape a cylinder: pollen tube as a model system for the generation of complex cellular geometry. Sex Plant Reprod 2010, 23:63-71.

14. Kato N, He H, Steger AP: A systems model of vesicle trafficking in Arabidopsis pollen tubes. Plant Physiol 2010, 152:590-601.

doi:10.1186/jbiol233

Cite this article as: Feijó JA: The mathematics of sexual attraction. Journal of Biology 2010, 9:18. 